\title{
OSCILLATION PROBLEM IN PITCH ANGLE CONTROL OF WINGED BODY
}

\author{
Igor Škrjanc* Marko Lepetić * Sašo Blažič * \\ Yasuhiro Morita ${ }^{* *}$ \\ * University of Ljubljana, Faculty of Electrical Engineering, \\ Tržaška 25, 1000 Ljubljana, Slovenia \\ ** Japan Aerospace Exploration Agency, Institute of Space \\ and Astronautical Science
}

\begin{abstract}
The requirements in pitch angle control of winged body are a very fast response with as less oscillation as possible. The oscillation can damage the equipments which are carried within the body. The main problem to cope with is in relatively fast and oscillatory dynamics of the rocket and slow actuators and sensors. We solve the problem by using predictive approach. The main idea of this approach is in process output prediction based on decomposed process model. The decomposition enables the extension of model-based approach to the processes with integrative behavior such as the rocket pitch angle control. The proposed approach gives a framework to design a control for a wide range of processes. In the paper the predictive design methodology was compared to the classical approach. The proposed approach gives a very fast response. Moreover, amplitude oscillation of the winged body are kept low. Copyright (C)2005 IFAC
\end{abstract}

Keywords: modelling, compensation, predictive control, decomposition methods, oscillation

\section{INTRODUCTION}

The requirements in pitch angle control of winged body are a very fast response with as less oscillation as possible. This is difficult task because a very fast and oscillatory dynamics of the body is combined with relatively slow actuators. There is another problem of not exactly known parameters of the process. The aerodynamic parameters are given in the range of \pm 40 percents. They depend on actual weather conditions. The change of those parameters causes a serious problem by the design

\footnotetext{
1 The work was done in the frame of bilateral project between Japan Society for the Promotion of Science and Ministry of Education, Science and Sport of the Republic of Slovenia, Attitude Control of the Winged Test Vehicle. The author would like to thank to both institutions for given support.
}

of control which should be robust in the whole range of parameter changes and should give an appropriate control performance.

The winged body is actually the rocket to test the rocket engines. The first approach to the pitch angle control was done by using a classical compensator. This lead to an appropriate response in the case of nominal parameters but for the wide range of parameter changes the responses are not satisfactory, especially because of high frequency oscillation.

The idea is to implement the model-based predictive control strategy similar to those presented by Kaya (2004) and Tan et al. (2003) . The basic idea which was proposed by Škrjanc and Matko (2000) is in this paper extended to the processes with integrative nature. In this case the model is 
decomposed into two parallel models and included into the design of control law. This modification enables the prediction of the future model output. The control algorithm is called a decomposedmodel predictive control (DMPC). The control law is than obtained on the basic principles of predictive control (Škrjanc and Matko, 2000). The main advantage of our approach is the fact that it offers the framework to design the control for a wide range of different processes: integrative, unstable, phase non-minimal, with time-delay, processes of higher order and multivariable processes. The problem of time-delay is solved as it is shown by Kaya (2004) and Lee (2000).

The paper is organized in the following way: in Section 2 the modelling of the rocket dynamics is given, in Section 3 the decomposed-model predictive control algorithm is described and in Section 4 the simulation study is given, where also the comparison with the classical compensator which was used before is presented.

\section{THE DYNAMICS OF THE ROCKET}

The rocket is modelled as a winged rigid body. Its shape and basic dimensions are given in Fig. 1. In our case only the control of longitudinal motion of the rocket will be treated, i.e. only the the model $\frac{\Theta(s)}{\delta(s)}$ will be investigated, where $\Theta$ is pitch angle and $\delta$ is control surface deflection angle . The diagram of the forces and torques is shown in Fig. 2 where $x$ and $z$ represent the coordinates of the system, $V$ is the airspeed, $u$ and $w$ are the

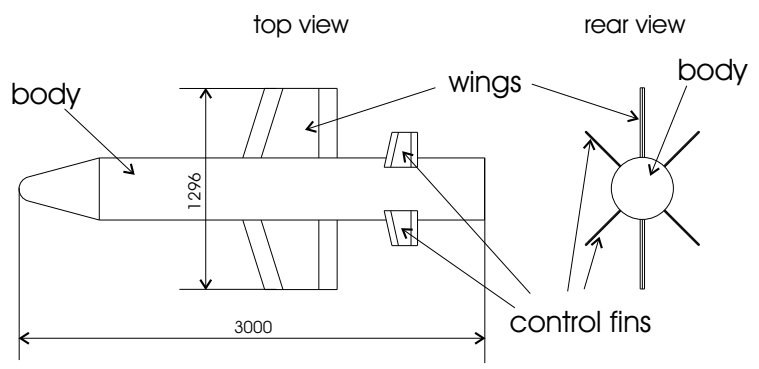

Fig. 1. The shape and basic dimension of the rocket.

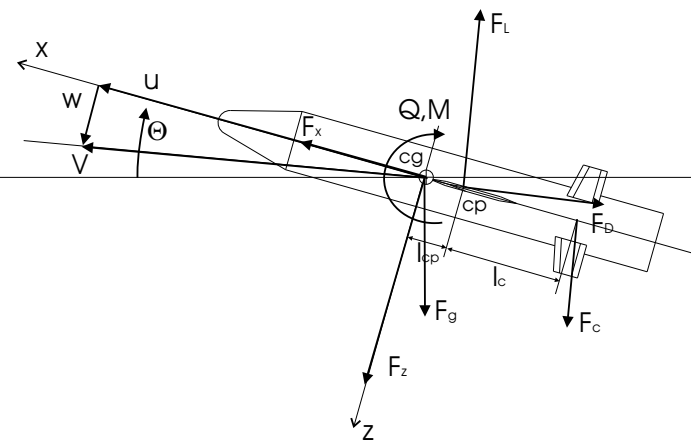

Fig. 2. The diagram of forces and torques. airspeed components in $x$ and $z$ coordinates, $\Theta$ is the pitch angle, $Q$ is the angular velocity, $F_{g}$ stands for the gravity force, $F_{L}$ is the lift force, $F_{D}$ the drag force, $F_{x}$ is the air pressure force in the direction of $x$ axis, $F_{z}$ is the air pressure force in the direction of $z$ coordinate, $M$ is the torque caused by lift and drag, $F_{c}$ stands for the control force, $c p$ is the center of pressure, $c g$ is the center of gravity, $l_{\mathrm{cp}}$ is the handle of pressure center and $l_{\mathrm{c}}$ is the handle of control force.

The motion of rigid body in all three dimensions can be represented by Euler equations of motion. For $2 \mathrm{D}$ problem the motion can be described with the following equations:

$$
\begin{array}{r}
F_{x}-m g \sin \Theta=m(\dot{u}+Q w) \\
F_{z}+m g \cos \Theta=m(\dot{w}-Q u) \\
M=I_{y} \dot{Q} \\
Q=\dot{\Theta}
\end{array}
$$

The airflow around the specific shape is difficult to describe using mathematical formulas, therefore the aerodynamic forces and moments are calculated using aerodynamic coefficients. For the winged body the following can be written:

$$
\begin{array}{r}
F_{L}=q S C_{L \alpha} \alpha \\
F_{D}=q S C_{D} \\
q=\frac{\rho V^{2}}{2} \\
\alpha=\arctan \frac{w+Q l_{c p}}{u}
\end{array}
$$

where $F_{L}$ and $F_{D}$ are the lift and drag forces, $S$ is reference area, $\alpha$ stands for angle of attack, and $q$ is the dynamical pressure. Aerodynamic coefficients $C_{L \alpha}$ and $C_{D}$ denote the lift coefficient and the drag coefficient, respectively. They are usually measured and given as a look-up table. Both coefficients varies in the range of about \pm 40 percent. This means that the implied control should have a great robustness. The $\mathrm{x}$ and $\mathrm{z}$ component of body force can be written for the geometry of Fig. 2:

$$
\begin{aligned}
F_{X B} & =-F_{D} \cos \alpha+F_{L} \sin \alpha \\
F_{Z B} & =-F_{L} \cos \alpha-F_{D} \sin \alpha
\end{aligned}
$$

The lift and drag forces cause also the torque. It could be calculated by multiplying these forces with the distance between the center of gravity and the center of pressure. This distance depends on the angle of attack $\alpha$. To calculate the torque of the body the following equation is used:

$$
M_{B}=q S l C_{M \alpha} \alpha
$$

where $l$ is the reference length and $C_{M \alpha}$ is another coefficient that stands for: 


$$
C_{M \alpha}=C_{L \alpha} \frac{l_{c p}}{l}
$$

and is also usually given in the look-up table. Deflection of the control surface for the angle $\delta$ results in the control force $F_{C}$ :

$$
F_{C}=q S C_{L \delta} \delta
$$

The shape of the rocket is rather unusual. Four tail fins are set in shape of letter $\mathrm{X}$ as shown in Fig. 1. The aileron, elevator and rudder (roll, pitch and yaw) effects are given with the combination of the tail fins deflections. The control torque is obtained multiplying the control force with the distance to the axis of rotation (the center of gravity). When the control surfaces work as an elevator the $\mathrm{x}$ and $\mathrm{z}$ component of the control force $\left(F_{X C}\right.$ and $\left.F_{Z C}\right)$ and the control torque $\left(M_{C}\right)$ are:

$$
\begin{array}{r}
F_{X C}=n_{e} F_{C} \sin \alpha \\
F_{Z C}=n_{e} F_{C} \cos \alpha \\
M_{C}=F_{Z C} l_{c}
\end{array}
$$

with $n_{e}=2 \sqrt{2}$. The common forces and torques of the body and control surfaces are:

$$
\begin{array}{r}
F_{X}=F_{X B}+F_{X C} \\
F_{Z}=F_{Y B}+F_{Z C} \\
M=M_{B}+M_{C}
\end{array}
$$

The nonlinear model of the rocket dynamics is described in Eq. (8). For the control purposes, this model can be linearized to obtain simpler linear model.

The model was linearized around the following stationary state: $u=75 \mathrm{~m} \mathrm{~s}^{-1}, w=0, \alpha=0$, $Q=0$ and $\Theta=0$. We get the following transfer function:

$$
G_{r b}(s)=\frac{\Omega(s)}{\delta_{a}(s)}=\frac{n_{e}\left(b_{1} s+b_{0}\right)}{\left(s^{2}+a_{1} s+a_{0}\right)}
$$

where $G_{r b}(s)$ stands for the rocket body transfer function between the pitch angle velocity $\Omega$ and the angle of the deflection fins $\delta_{a}$ and $n_{e}, a_{1}, a_{0}$, $b_{1}$ and $b_{0}$ stand for:

$$
\begin{array}{r}
a_{1}=\frac{q S C_{L \alpha}}{m u}+\frac{q S C_{M \alpha} l l_{c p}}{I_{y} u} \\
a_{0}=-\frac{q S C_{M \alpha} l}{I_{y}} \\
b_{1}=\frac{q S C_{L \alpha} l_{c}}{I_{y}} \\
b_{0}=\frac{q^{2} S^{2} C_{L \delta}}{m u I_{y}}\left(C_{L \alpha} l_{c}+C_{M \alpha} l\right)
\end{array}
$$

with the following values of parameters at the point of linearization: $n_{e}=2 \sqrt{2}, l=3.386 \mathrm{~m}$, $m=260 \mathrm{~kg}, S=0.12566 \mathrm{~m}^{2}, I_{y}=253.1 \mathrm{~kg}$ $\mathrm{m}^{2}, C_{M \alpha}=-0.6393, C_{L \alpha}=23.5, C_{L \delta}=3.0197$, $l_{c}=-0.8285 \mathrm{~m}, l_{c p}=-0.0921 \mathrm{~m}$.

Another problem is caused by the constraints of the deflection fins in the range of $-0.05 \mathrm{rad}$ $\leq \delta_{a} \leq 0.05 \mathrm{rad}$ and the dynamics of the angle velocity sensor. The transfer functions of both are given next:

$$
\begin{aligned}
& G_{a}=\frac{\delta_{a}(s)}{\delta(s)}=\frac{\omega_{1}^{2}}{s^{2}+2 \zeta_{1} \omega_{1} s+\omega_{1}^{2}} \frac{\tau_{a_{1}} s+1}{\tau_{a_{2}} s+1} \\
& G_{s}=\frac{\Omega_{s}(s)}{\Omega(s)}=\frac{\omega_{2}^{2}}{s^{2}+2 \zeta_{2} \omega_{2} s+\omega_{2}^{2}} \frac{-\tau s+1}{\tau s+1}
\end{aligned}
$$

where $\omega_{1}=28 \pi \mathrm{rad} \mathrm{s}^{-1}, \zeta_{1}=0.5, \frac{1}{\tau_{a_{1}}}=40 \pi \mathrm{s}^{-1}$, $\frac{1}{\tau_{a_{2}}}=5 \pi \mathrm{s}^{-1}$, and $\omega_{2}=54 \pi \mathrm{rad} \mathrm{s}{ }^{-1}, \zeta_{2}=0.5$, $\tau=0.0085 \mathrm{~s}$.

Taking into account the rocket dynamics, the actuator and the sensor, the dynamics of the whole process is the following:

$$
G_{r}(s)=\frac{\Omega_{s}(s)}{\delta(s)}=G_{a}(s) G_{r b}(s) G_{s}(s) e^{-s T_{d}}
$$

where $T_{d}$ stands for the time-delay which is due to the computational time and is equal to $T_{d}=0.015$ s.

The frequency response of the whole process is shown in Fig. 3. The small resonance frequency pick of the rocket system appears at frequency of $2.1 \mathrm{rad} / \mathrm{s}$. In Fig. 4 the step response of the openloop system is given where the high-frequency oscillations can be observed.
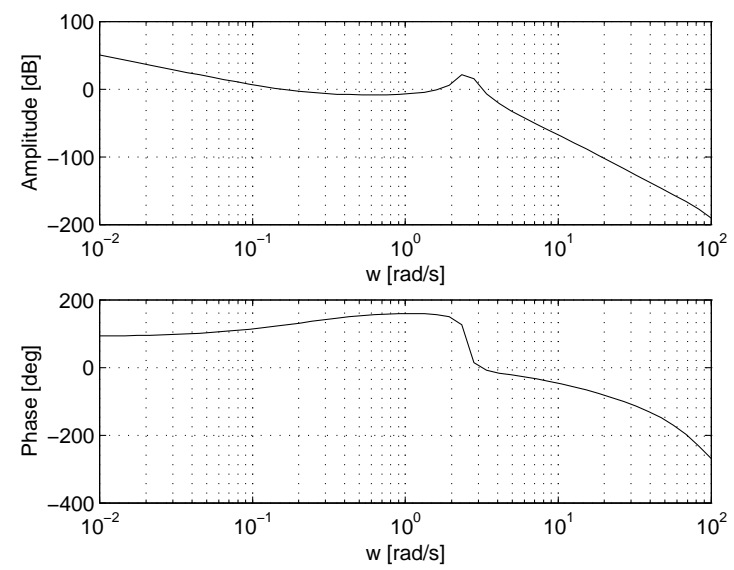

Fig. 3. The frequency response of the rocket system.

\section{DECOMPOSED-MODEL PREDICTIVE CONTROL}

Model-based predictive formulation for control of open-loop unstable processes is frequently used as an optimization control problem (Nagrath, 2002). 


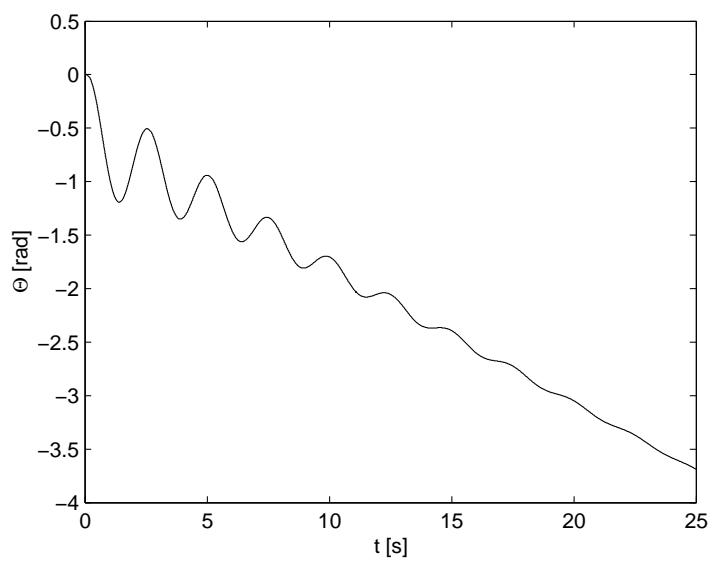

Fig. 4. The step response of the rocket system.

In the case of fast and open-loop unstable processes this approach can not be implemented. The realization requirements demand a computation algorithm which can be calculated very fast not to enlarge the computation time delay. This means that the control algorithm should be given in analytical way.

In this section the main idea of decomposed model predictive control will be given. And it is given in discrete-time domain which is the most natural for predictive techniques. Let the process model be the following:

$$
\begin{array}{r}
x_{m}(k+1)=\mathbf{A}_{m} x_{m}(k)+\mathbf{B}_{m} u(k) \\
y_{m}(k)=\mathbf{C}_{m} x_{m}(k)
\end{array}
$$

where $\mathbf{A}_{m}, \mathbf{B}_{m}$ and $\mathbf{C}_{m}$ are the time-discrete state-space matrices of the process model without taking into account the process delay.

The main idea of predictive algorithm (Škrjanc and Matko, 2000) is to determine the future control action so that the predicted output value coincide with the reference trajectory. The point where the reference and output signal coincide is called a coincidence horizon and denoted by $H$. The prediction is calculated under assumption of constant future manipulated variables $(u(k)=$ $u(k+1)=\ldots=u(k+H-1))$, i.e. the mean level control. Under those assumption the $H$-step ahead prediction of the model output at time instant $k$ can be easily obtained as follows:

$$
y_{m}(k+H \mid k)=\mathbf{C}_{m}\left(\mathbf{A}_{m}^{H} \mathbf{x}_{m}(k)+\mathbf{K} u(k)\right)
$$

with $\mathbf{K}=\left(\mathbf{A}_{m}^{H}-\mathbf{I}\right)\left(\mathbf{A}_{m}-\mathbf{I}\right)^{-1} \mathbf{B}_{m}$. When the system matrix $\mathbf{A}_{m}$ is Hurwitz, than the predictive control law can be calculated using prediction in Eq. (14). In the case of integrative behavior the system matrix $\mathbf{A}_{m}$ is not Hurwitz (Braatz, 1996) and the proposed prediction cannot be applied. In this case the process model transfer function should be decomposed in the following way:

$$
G_{m}(z)=G_{m_{p}}(z)+G_{m_{i}}(z)
$$

where $G_{m_{p}}(z)$ and $G_{m_{i}}(z)$ stand for proportional and pure integrative part.

The state-space equivalent of the transfer function $G_{m_{p}}(z)$ is described by matrices $\mathbf{A}_{m_{1}}, \mathbf{B}_{m_{1}}$ and $\mathbf{C}_{m_{1}}$ and the state-space equivalent $\mathbf{A}_{m_{2}}, \mathbf{B}_{m_{2}}$ and $\mathbf{C}_{m_{2}}$ for the transfer function $G_{m_{i}}(z)$. The prediction of process model output is calculated as sum of both predictions

$$
\begin{aligned}
& y_{m_{1}}(k+H)=\mathbf{C}_{m_{1}}\left(\mathbf{A}_{m_{1}}^{H} \mathbf{x}_{m_{1}}(k)+\mathbf{K}_{m} u(k)\right) \\
& y_{m_{2}}(k+H)=\mathbf{C}_{m_{2}}\left(\mathbf{x}_{m_{2}}(k)+H \mathbf{B}_{m_{2}}\right) u(k)
\end{aligned}
$$

with $\mathbf{K}_{m}=\left(\mathbf{A}_{m_{1}}^{H}-\mathbf{I}\right)\left(\mathbf{A}_{m_{1}}-\mathbf{I}\right)^{-1} \mathbf{B}_{m_{1}}$ and is given as follows

$$
y_{m}(k+H)=y_{m_{1}}(k+H)+y_{m_{2}}(k+H)
$$

The behavior of the closed-loop system in the case of proposed predictive control technique is defined by the reference trajectory given by referencemodel transfer function. The reference-model trajectory is given implicitly by exponential factor which describes how the control error should behave in the future. Through this exponential factor, which is analog to the the time constant of the reference model, we will predict the control error for $H$-step ahead, at the so called coincidence horizon as given next:

$$
w(k+H)-y_{p}(k+H)=a_{r}^{H} \cdot\left(w(k)-y_{p}(k)\right)
$$

where $w(k)$ and $y_{p}(k)$ stands for the current reference signal and output signal of the process and $a_{r}$ denotes the pole of the reference model. Assuming the equivalence of the predicted reference model trajectory and the estimated process output at the coincidence horizon and constant reference signal in the future, the reference trajectory or desired dynamics of the process output is given implicitly as follows:

$$
y_{p}(k+H)=w(k)-a_{r}^{H} \cdot\left(w(k)-y_{p}(k)\right)
$$

where $a_{r}$ implicitly describes the reference-model trajectory.

The main goal of the proposed algorithm is to find the control law which enables the reference trajectory tracking of the controlled signal. In other words, $u(k)$ has to be found to fulfill Eq. (17). The estimated value of the process output is given as

$$
y_{p}(k+H)=y_{p}(k)+y_{m}(k+H)-y_{m}(k)
$$

It is obtained under assumption that the plant output will change for the same amount as its model in the same interval of time. 
Combining Eq. (17), (18) and the prediction of model output given in Eq. (16), the following is obtained:

$$
\begin{array}{r}
u(k)=g_{0}^{-1}\left(\left(1-a_{r}^{H}\right)\left(w(k)-y_{p}(k)\right)+\mathbf{F} \mathbf{x}_{m_{1}}(k)\right) \\
\mathbf{F}=\mathbf{C}_{m_{1}}\left(\mathbf{I}-\mathbf{A}_{m_{1}}^{H}\right)
\end{array}
$$

where $g_{0}$ stands for:

$$
\begin{array}{r}
g_{0}=\mathbf{C}_{m_{1}}\left(\mathbf{A}_{m_{1}}^{H}-\mathbf{I}\right)\left(\mathbf{A}_{m_{1}}-\mathbf{I}\right)^{-1} \mathbf{B}_{m_{1}}+ \\
+H \mathbf{C}_{m_{2}} \mathbf{B}_{m_{2}}
\end{array}
$$

The control law in Eq. 19 is valid for the process without time-delay. When we are dealing with time-delayed processes than the undelayed process output should be estimated. The estimation of process output $\hat{y}_{p}(k)$ can be done based on the current process output and the delayed and undelayed model outputs as given next

$$
\hat{y}_{p}(k)=y_{p}(k)-y_{m}^{d}(k)+y_{m}(k)
$$

where $y_{m}^{d}(k)$ stands for time-delayed process model and $y_{m}(k)$ for undelayed model.

The control law of DMPC in analytical form can be on the other way presented as:

$$
\begin{array}{r}
u(k)=K_{d}\left(w(k)-y_{p}^{0}(k)\right)+K_{x_{m_{1}}} \mathbf{x}_{m_{1}}^{0}(k) \\
K_{d}=g_{0}^{-1}\left(1-a_{r}^{H}\right) \\
K_{x_{m_{1}}}=g_{0}^{-1} \mathbf{C}_{m_{1}}\left(\mathbf{I}-\mathbf{A}_{m_{1}}^{H}\right)
\end{array}
$$

Note that the DMPC control law is realizable if the gain $g_{0}$ is non-zero. This is true if $H \geq \rho$, where $\rho$ is the relative order of the system.

\subsection{The pitch angle control using DMPC}

When we design the DMPC for pitch angle control of the rocket only the body dynamics was taken into account. The transfer function $\frac{\Theta(s)}{\delta_{a}(s)}$ should be decomposed into a parallel decomposition. In general this means that the systems with transfer function $\frac{B(s)}{s A(s)}$ where $A(s) \neq 0$ for $s=0$ should be decomposed into

$$
\frac{B(s)}{s A(s)}=\frac{Q(s)}{A(s)}+\frac{K}{s}=G_{m_{p}}(s)+G_{m_{i}}(s)
$$

where $A(s)=a_{n} s^{n}+a_{n-1} s^{n-1}+\ldots+a_{0}$ and $B(s)=b_{n} s^{n}+b_{n-1} s^{n-1}+\ldots+b_{0}$.

The solution of Diofantine equation in Eq. 21 is the following:

$$
\begin{array}{r}
K a_{0}=b_{0} \\
q_{i}+K a_{i+1}=b_{i+1}, \quad i=0, \ldots, n-1
\end{array}
$$

where $Q(s)=q_{n-1} s^{n-1}+q_{n-2} s^{n-2}+\ldots+q_{0}$.
After decomposition of the continuous transfer function into the parallel form both transfer functions $G_{m_{p}}(s)$ and $G_{m_{i}}(s)$ are transformed to the discrete-time state-space presentation. This enables the calculation of DMPC parameters. To have a suitable performance and appropriate robustness of the control in this case, the DMPC was tuned with the following parameters: $T_{r}=7$ $\mathrm{s}$ and $H=5$. The algorithm was implemented using the sampling time of $T_{s}=0.01 \mathrm{~s}$. The tuning parameters result in the control parameters $K_{d}=-0.9297$ and $K_{x_{m_{1}}}=[-0.3959,0.3967]$.

\section{SIMULATION STUDY}

The proposed DMPC approach was tested by simulation and compared with simple compensator which is designed using optimization. The goal of the attitude control is to control the pitch angle $\Theta$ by the manipulated variable $\delta_{a}$ to have the fastest response with as less oscillation as possible which can cause the serious problems.

In the case of compensator the following control law is obtained:

$$
\delta(s)=K_{p}\left(\Theta_{r}(s)-\Theta(s)\right)-K_{r} \cdot G_{f}(s) \Omega_{s}(s)
$$

where $\Theta_{r}(s)$ stands for Laplace transform of the reference signal, $K_{p}=-2.2383, K_{r}=-0.7967$ and $G_{f}(s)$ stands for the filter of the pitch angle velocity

$$
G_{f}(s)=\frac{\omega_{f}^{2}}{s^{2}+2 \zeta_{f} \omega_{f} s+\omega_{s}^{2}}
$$

where $\omega_{f}=20 \pi \mathrm{rad} \mathrm{s}^{-1}$ and $\zeta_{f}=0.8$.

The control results and comparison of both control algorithms for the nominal values of system parameters are shown in Fig. 5.
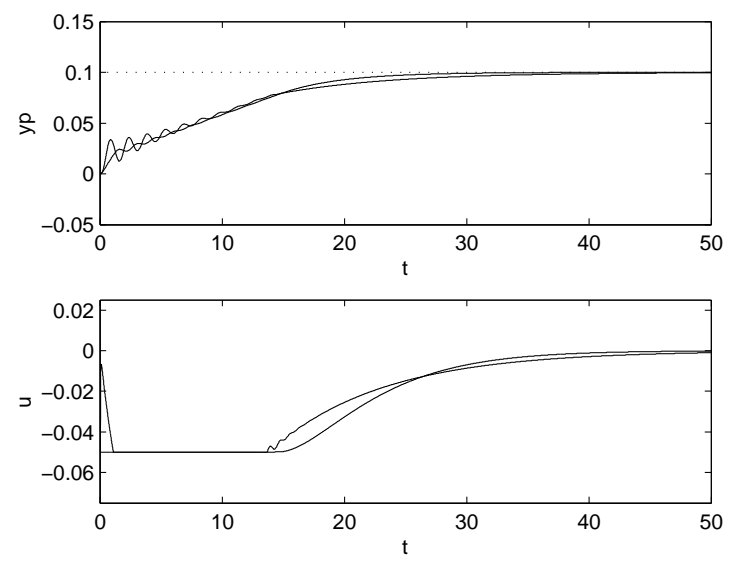

Fig. 5. The comparison between simple compensation control and DMPC control.

The compensator approach shows very oscillatory response at the beginning and it is also slightly 

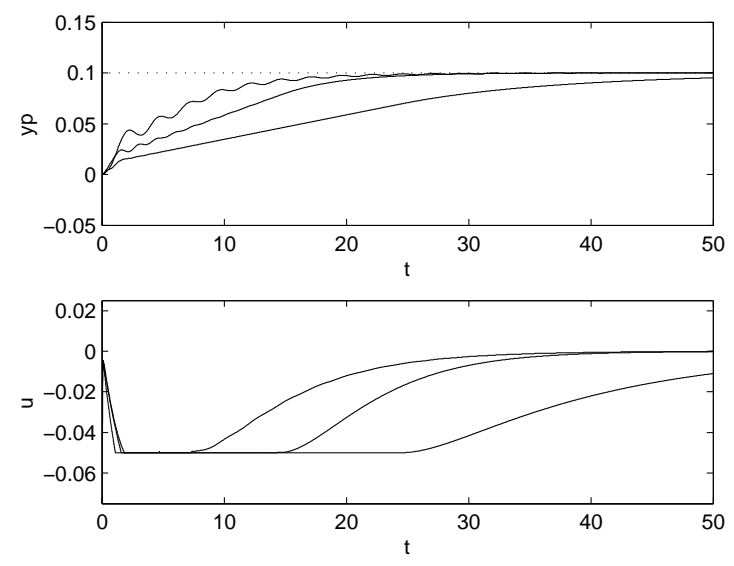

Fig. 6. The DMPC control for different values of system parameters.

slower. The DMPC control law results in faster response and smaller oscillation. Both control algorithms do not reject the input disturbance, but this is actually not necessary in the case of attitude control.

The robustness in our case is of great importance because the aerodynamic coefficients $C_{L \alpha}$ and $C_{L \delta}$ vary in the range of about \pm 40 percent from nominal value $\left(C_{L \alpha}=C_{L \alpha}^{n}(1 \pm 0.4), \quad C_{L \delta}=\right.$ $\left.C_{L \delta}^{n}(1 \pm 0.4)\right)$, where $C_{L \alpha}^{n}$ and $C_{L \delta}^{n}$ stand for the nominal values. We have simulated the closed loop behavior where the controller was designed for the nominal values of parameters and the process parameters have changed in the proposed range. All three responses, for the nominal case of process parameters, for the positive deviation of parameters and for the negative deviation of parameters have been investigated.

In Fig. 6 we have shown the influence of the system parameter changes to the performance of the DMPC control algorithm. It is shown that the close-loop responses in the case of process parameter mismatch satisfy the normal performance requirements.

The same robustness test was performed also for the compensator case as shown in Fig. 7. The obtained results show that the control is less robust than in the case of DMPC in spite of the fact that nominal response of DMPC is slightly faster than in the compensator case.

\section{CONCLUSION}

In the paper the decomposed-model predictive control was implemented to control the pitch angle of the rocket. The proposed control approach is not limited to this type of processes, but it can be implemented to a wide range of different processes. The main advantages of the proposed approach is a very simple design which results in
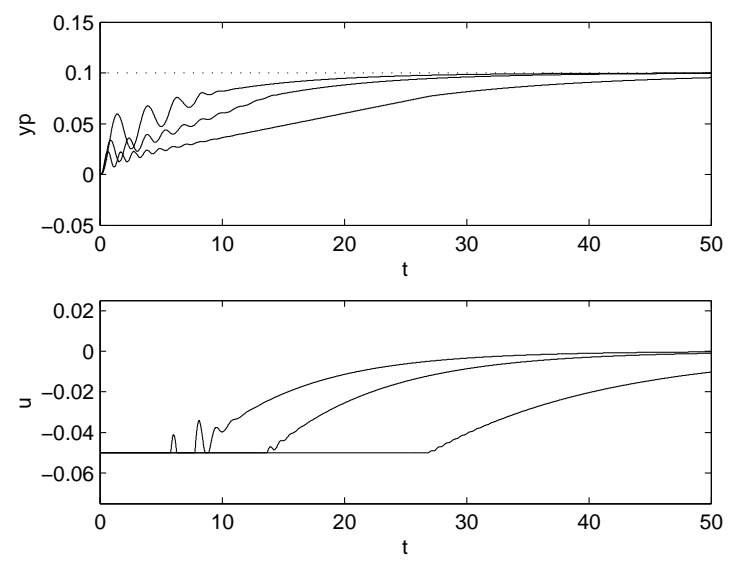

Fig. 7. The compansation control for different values of system parameters.

better performance as proposed before. To show the potential use of the proposed approach, we made the comparison with classical compensator control which is known approach to control the pitch angle of the rocket. The robust performance of the proposed approach was also examined by simulation and it has been shown that the proposed approach offers higher robustness and better performance than the classical approach.

\section{REFERENCES}

Braatz, R. D. (1996). Internal model control. In: Control Handbook. pp. 215-224. Butterworths, CRC Press.

Kaya, I. (2004). Imc based automatic tuning method for pid controllers in a smith predictor configuration. Computers and Chemical Engng. 28, 281-290.

Lee, Y., Lee J. Park S. (2000). Pid controller tuning for integrating and unstable processes with time delay. Chem. Engng. Science 55, 3481-3493.

Nagrath, D., Prasad V. Bequette B. W. (2002). A model predictive formulation for open-loop unstable cascade systems. Chem. Engng. Science 57, 365-378.

Tan, W., H. J. Marquez and T. Chen (2003). Imc design for unstable processes with time delays. Journal of Process Control 13, 203213.

Škrjanc, I. and D. Matko (2000). Predictive functional control based on fuzzy model for heatexchanger pilot plant. IEEE Transection on Fuzzy Systems 8(6), 705-712. 